\title{
Uso de morfina, xilazina e meloxicam para o controle da dor pós-operatória em cadelas submetidas à ovariossalpingo-histerectomia
}

\author{
[Morphine, xylazine and meloxicam in pain management after ovariosalpingohysterectomy of bitches] \\ D.A. Pereira ${ }^{1}$, J.A. Marques ${ }^{2 *}$ \\ ${ }^{1}$ Aluna de pós-graduação - FCAV-UNESP - Jaboticabal, SP \\ ${ }^{2}$ Faculdade de Ciências Agrárias e Veterinárias - UNESP - Jaboticabal, SP \\ Via de Acesso Prof. Paulo Donato Castellane s/n \\ 14884-900 - Jaboticabal, SP
}

\begin{abstract}
RESUMO
Foram realizados estudos empregando-se analgésicos por via epidural e subcutânea em cadelas de diferentes raças e idades, submetidas à castração mediante celiotomia. Vinte animais foram tranquilizados e anestesiados com tiletamina-zolazepam, e aleatoriamente distribuídos em quatro grupos $(n=5)$, de acordo com o fármaco e a via de administração. Os do grupo morfina (GM) foram submetidos à anestesia epidural no espaço lombossacro, com morfina $(0,1 \mathrm{mg} / \mathrm{kg})$ associada ao cloreto de sódio a $0,9 \%$. Aos do grupo xilazina (GX), foram administrados xilazina $(0,2 \mathrm{mg} / \mathrm{kg})$ e cloreto de sódio a $0,9 \%$. Os do grupo meloxicam (GME) receberam $0,2 \mathrm{mg} / \mathrm{kg}$ do anti-inflamatório meloxicam associado ao cloreto de sódio a $0,9 \%$, injetado pela via subcutânea. Os do grupo-controle (CG) receberam apenas cloreto de sódio a $0,9 \%$. O volume final para as injeções epidurais foi padronizado para $0,3 \mathrm{~mL} / \mathrm{kg}$. A mensuração inicial da concentração de cortisol plasmático, do ritmo cardíaco, da frequência respiratória e os parâmetros comportamentais foram registrados imediatamente antes do procedimento cirúrgico (M1). Registros adicionais foram apresentados às 2, 6, 12 e 24 horas após o procedimento cirúrgico (M2, M3, M4 e M5, respectivamente). As variáveis comportamentais foram avaliadas por meio de sinais clínicos e seus respectivos escores. Em GX foram observadas depressão respiratória, bradicardia e concentração de cortisol mais alta do que o registrado no GM. A analgesia obtida pelo meloxicam foi considerada ineficiente. É possível concluir que a morfina, via epidural, promoveu menor incidência de efeitos colaterais e melhor analgesia e bem-estar animal.
\end{abstract}

Palavras-chave: cão, opioides, alfa-2-agonistas, meloxicam

\begin{abstract}
The use of analgesics by epidural and subcutaneous way in bitches submitted to surgical sterilization by laparotomy was evaluated. Twenty females dogs of different ages and breeds were sedated and anesthetized with a combination of tiletamine-zolazepam and randomly distributed into four experimental groups of five animals each. Through the epidural space, the animals of each group received $0.1 \mathrm{mg} / \mathrm{kg}$ of morphine (MG) or $0.2 \mathrm{mg} / \mathrm{kg}$ of xylazine $(X G)$; while the control group $(C G)$ received a $0.9 \%$ solution of chloride sodium. The final volume stabilished for lumbosacral epidural injections was $0.3 \mathrm{~mL} / \mathrm{kg}$. The dogs of meloxicam group (MEG) subcutaneously received $0.2 \mathrm{mg} / \mathrm{kg}$ of the drug. The initial measurement of plasmatic cortisol concentration, heart and respiratory rates, and behaviour parameters were evaluated as soon as the surgical procedure had finished (M1). These evaluations were repeated after 2, 6, 12, and 24 hours of the postoperative period (M2, M3, M4, and M5 respectively). The behaviour parameters were evaluated by clinical signs and respective scores. Values of cortisol concentration were higher in the xylazine group than the ones registered in the morphine group. Meloxicam failed to provide pain control. Epidural use of morphine guaranteed fewer side effects and better pain control.
\end{abstract}

Keywords: dog, opioids, alfa-2-agonists, meloxicam

Recebido em 27 de maio de 2008

Aceito em 23 de fevereiro de 2009

* Autor para correspondência (corresponding autor)

E-mail: jmarques@fcav.unesp.br 


\section{INTRODUÇÃO}

A associação de fármacos é recomendada para vários procedimentos cirúrgicos, incluindo-se as cirurgias eletivas como a ovariossalpingohisterectomia em cadelas, empregada no controle populacional de cães errantes. Silva (1997) relatou que, dentre as técnicas regionais, há a anestesia espinhal, que é classificada em peridural e subaracnoidea, sendo a técnica epidural em pequenos animais largamente empregada na rotina clínica.

Silva (1997) e Fierheller et al. (2004) citaram a utilização da técnica epidural na analgesia intra e pós-operatória dos animais domésticos. Essa técnica anestésica oferece vantagens principalmente por ser de fácil execução e baixo custo, permitir a realização de bloqueios diferenciados para obter maior período de ação, o que depende da dose e da concentração do anestésico local e/ou analgésico utilizado, e não exigir aparelhagem sofisticada.

A prevenção e o controle da dor são o tema central da prática anestésica. É essencial que o anestesista tenha um entendimento dos processos fisiológicos que conduzem à percepção da dor e as respostas do paciente a este estímulo doloroso. A dor é descrita por Thurmon et al. (1996) como uma experiência emocional e sensorial desagradável associada a uma lesão tissular real e potencial, ou é descrita em termos de um dano propriamente dito.

Segundo Oleskovicz (2001), o processo doloroso inicia-se na periferia, a partir de uma lesão tissular, após a ativação de receptores especializados na condução do impulso nociceptivo, que transmitem a mensagem nociceptiva à medula espinhal através de fibras nervosas sensoriais, ou seja, fibras aferentes primárias. A dor primária é transmitida pelas fibras dolorosas tipo A $\delta$, que são mielinizadas, e a dor secundária por estimulação das fibras do tipo $\mathrm{C}$, que são amielinizadas. Os mecanismos que processam as informações nociceptivas espinhal e supraespinhal são interrompidos ou minimizados, por meio da aplicação espinhal de analgésicos ou anestésicos. Assim, a técnica epidural tem sido proposta como alternativa para o bloqueio da sensibilização central à dor, exercendo efeito negativo sobre a informação nociceptiva, produzindo analgesia.
Em geral, os benefícios do tratamento da dor superam os riscos associados à administração da substância analgésica, portanto médicos e médicos veterinários devem conscientizar-se de que a dor é um fator biológico ativo que pode causar consequências maléficas ao animal (Fantoni e Cortopassi, 2002).

Os objetivos destes estudos foram avaliar o bem estar animal pela redução da dor e do estresse no pós-operatório, observar o tempo e a duração da analgesia dos fármacos em cadelas submetidas à ovariossalpingo-histerectomia, e verificar os efeitos comportamentais, a concentração plasmática do cortisol e as variações dos parâmetros fisiológicos dos pacientes utilizandose quatro técnicas de controle da dor no pósoperatório, por meio da administração de fármacos pela via peridural.

\section{MATERIAL E MÉTODOS}

Foram utilizadas 20 cadelas hígidas, sem raça definida, originárias do município de Jaboticabal. Os animais foram distribuídos em quatro grupos experimentais, com cinco cadelas por grupo.

No primeiro grupo (GM; $n=5)$, as cadelas receberam sulfato de morfina ${ }^{1}$ associada à solução de cloreto de sódio a $0.9 \%{ }^{2}$ por via epidural, na dose de $0,1 \mathrm{mg} / \mathrm{kg}$; no segundo grupo $(\mathrm{Gx} ; \mathrm{n}=5)$, receberam cloridrato de xilazina ${ }^{3}$ associada à solução de cloreto de sódio a $0,9 \%$ pela técnica epidural, na dose de $0,2 \mathrm{mg} / \mathrm{kg}$; no terceiro (GME), receberam anti-inflamatório meloxicam $^{4}$ pela via subcutânea (SC), na dose de $0,2 \mathrm{mg} / \mathrm{kg}$ e solução fisiológica de cloreto de sódio a $0,9 \%$ pela via epidural; o quarto grupo de animais $(\mathrm{GC} ; \mathrm{n}=5)$ recebeu solução de cloreto de sódio a $0,9 \%$ pela via epidural. O cálculo do volume total para a injeção epidural foi de $0,3 \mathrm{~mL} / \mathrm{kg}$.

Os pacientes foram avaliados clinicamente antes da realização do procedimento cirúrgico. As cadelas foram castradas empregando-se $\mathrm{o}$ seguinte protocolo anestésico: medicação préanestésica - foi utilizada a acepromazina ${ }^{5}$, na

${ }^{1}$ União Quimíca Farmacêutica Nacional S.A. - São Paulo,

Brasil.

${ }^{2}$ Laboratório Sanobiol Ltda - São Paulo, Brasil.

${ }^{3}$ Bayer S.A - São Paulo, Brasil.

${ }^{4}$ Boehringer Ingelheim do Brasil - São Paulo, Brasil.

${ }^{5}$ Univet S.A- São Paulo, Brasil. 
dose de $0,1 \mathrm{mg} / \mathrm{kg}$ pela via intramuscular; a indução e a manutenção foram feitas empregando-se tiletamina-zolazepam ${ }^{6}$, na dose de $10 \mathrm{mg} / \mathrm{kg}$ por via intramuscular. As cadelas receberam fluidoterapia de acordo com o cálculo de $10 \mathrm{~mL} / \mathrm{kg} / \mathrm{h}$ de solução fisiológica a $0,9 \%$. Após o término da cirurgia, todos os animais receberam enrofloxacina ${ }^{3}$ por via subcutânea, na dose de $5 \mathrm{mg} / \mathrm{kg}$. O procedimento cirúrgico teve uma duração variável de 1 a 1,5 horas. Os pontos da ferida cirúrgica e o tamanho da incisão foram padronizados.

Nos quatro grupos, a injeção das soluções foi realizada no espaço epidural lombossacro, sendo identificada pela palpação das tuberosidades isquiáticas, associadas à palpação da depressão entre as vértebras. Foram realizados todos os procedimentos necessários para a punção do canal espinhal com a antissepsia da região previamente tricotomizada com iodopovidine.

Após a localização do espaço epidural, foi introduzida uma agulha $40 \times 12^{7}$ no espaço, até atingir o canal espinhal. Confirmada a posição correta da agulha, o cateter epidural (16G) foi introduzido através da agulha no espaço lombossacro, direcionando-o cranialmente dentro do canal vertebral. Posteriormente a agulha hipodérmica foi retirada, sendo o cateter fixado na pele com fio náilon $2-0^{8}$. O cateter foi introduzido de $3-5 \mathrm{~cm}$ no interior do canal vertebral. A técnica epidural foi realizada após o procedimento cirúrgico. $\mathrm{O}$ registro dos parâmetros basais dos pacientes foi caracterizado nos tempos zero, antes do procedimento cirúrgico (M1), e às 2, 6, 12 e 24 horas após o procedimento cirúrgico (M2, M3, M4 e M5, respectivamente) e administração epidural dos fármacos.

Os parâmetros de frequência cardíaca, respiratória e temperatura retal foram aferidos e registrados nos tempos citados acima. A frequência cardíaca foi aferida utilizando oxímetro de pulso ${ }^{9}$, com o conjunto emissor e sensor posicionado na língua do animal. Os parâmetros de frequência respiratória foram aferidos mediante observação da movimentação

\footnotetext{
${ }^{6}$ Laboratório Virbac - S.A- São Paulo, Brasil.

${ }^{7}$ Indústria Cirúrgica Becton Dickinson - Curitiba, Brasil.

${ }^{8}$ Brasmédica - São Paulo, Brasil.

${ }^{9}$ Equipamentos Hospitalares Ltda - Rio de Janeiro, Brasil.
}

do gradil costal; a temperatura retal foi aferida com termômetro clínico introduzido na ampola retal durante um minuto.

Com relação ao cortisol plasmático, foram colhidos $3 \mathrm{ml}$ de sangue da veia jugular ou cefálica dos animais, para a dosagem das concentrações plasmáticas de cortisol. As amostras foram colocadas em tubos livres de anticoagulantes, centrifugados durante 10 minutos, em centrífuga ${ }^{10}$ a $1.048,13$ giros. A seguir as amostras do plasma foram colocadas em tubos tipo Eppendorf ${ }^{11}$, identificadas $\mathrm{e}$ congeladas a $-20^{\circ}$ C. As amostras foram analisadas pelo método de radioimunoensaio ${ }^{12}$ para a mensuração da concentração de cortisol. As colheitas foram realizadas durante os períodos de observação acima descritos.

Foi realizada a avaliação comportamental dos animais por meio dos sinais clínicos e de seus respectivos escores (Tab. 1), segundo Firth e Haldane (1999).

A análise estatística foi realizada com o uso do software SAS (Der e Everitty, 2002). Os dados referentes à frequência cardíaca, temperatura retal e frequência respiratória, e à dosagem do hormônio cortisol foram aferidos, tabelados e submetidos à análise de variância de medidas repetidas. Para comparação entre médias, foi usado o Teste Tuckey e significância de $\mathrm{P}<0,05$. Os dados relacionados ao escore comportamental foram submetidos ao teste $\mathrm{t}$, segundo o programa computacional SAS/2004.

\section{RESULTADOS E DISCUSSÃO}

Um a três (20 a 60\%) dos animais do GX apresentaram emêse e bradicardia aos 30 e 60 minutos após a administração do fármaco via epidural. A perda dos reflexos nervosos, ou sensitivos, dos membros pélvicos foi observada em três $(60 \%)$ cadelas, com duração máxima de 60 minutos.

\footnotetext{
${ }^{10}$ Fanem Ltda. - São Paulo, Brasil.

${ }^{11}$ Alfa Mare - Instrumentos Científicos e Médicos Ltda Uberaba, Brasil

${ }^{12}$ Coat-A-Count Cortisol - TKCO2 - Diagnostic Products Corporation - Los Angeles, EUA.
} 


\section{Pereira e Marques}

Tabela 1. Escala de escore para determinação do comportamento de cadelas hígidas

\begin{tabular}{|c|c|c|c|c|}
\hline & & $\begin{array}{c}\text { Escore de } \\
\text { comportamento }\end{array}$ & & \\
\hline $\begin{array}{l}\text { Resposta } \\
\text { manipulação }\end{array}$ & $\begin{array}{c}\text { Escore } 0 \\
\text { sem resposta }\end{array}$ & $\begin{array}{c}\text { Escore } 1 \\
\text { resposta mínima, } \\
\text { olha e se move }\end{array}$ & $\begin{array}{c}\text { Escore } 2 \\
\text { vira cabeça ferida, } \\
\text { leve vocalização }\end{array}$ & $\begin{array}{c}\text { Escore } 3 \\
\text { vira cabeça, intenção de } \\
\text { morder, severa } \\
\text { vocalização }\end{array}$ \\
\hline Alerta & $\begin{array}{c}\text { Escore } 2 \\
\text { permanece alheio ao } \\
\text { ambiente }\end{array}$ & $\begin{array}{c}\text { Escore } 3 \\
\text { desperta após } \\
\text { barulho }\end{array}$ & $\begin{array}{c}\text { Escore } 4 \\
\text { alerta máximo }\end{array}$ & \\
\hline Postura & $\begin{array}{l}\text { Escore } 5 \\
\text { sonolento, decúbito } \\
\text { esternal ou lateral, } \\
\text { responde ao } \\
\text { chamado }\end{array}$ & $\begin{array}{c}\text { Escore } 6 \\
\text { sonolento, cabeça } \\
\text { baixa, não } \\
\text { responde ao } \\
\text { chamado }\end{array}$ & $\begin{array}{c}\text { Escore } 7 \\
\text { animal em pé e alerta }\end{array}$ & \\
\hline Alimentação & $\begin{array}{c}\text { Escore } 7 \\
\text { não come nem bebe }\end{array}$ & $\begin{array}{c}\text { Escore } 8 \\
\text { resiste ao se } \\
\text { oferecer comida }\end{array}$ & $\begin{array}{c}\text { Escore } 9 \\
\text { come e bebe } \\
\text { normalmente }\end{array}$ & \\
\hline
\end{tabular}

$\begin{array}{lcc}\begin{array}{l}\text { Funções } \\ \text { fisiológicas }\end{array} & \begin{array}{c}\text { Escore 1 } \\ \text { urina e/ou defeca }\end{array} & \begin{array}{c}\text { Escore 2 } \\ \text { não urina nem } \\ \text { defeca }\end{array} \\ \text { Temperatura } & \begin{array}{c}\text { Escore } 0 \\ \text { normal }\end{array} & \begin{array}{c}\text { Escore 1 } \\ \text { anormal }\end{array} \\ \text { Salivação } & \begin{array}{c}\text { Escore } 0 \\ \text { normal }\end{array} & \begin{array}{c}\text { Escore 1 } \\ \text { anormal }\end{array} \\ \text { Aparência } & \begin{array}{c}\text { Escore 0 } \\ \text { normal }\end{array} & \begin{array}{c}\text { Escore 1 } \\ \text { pálpebras } \\ \text { fechadas, orelhas } \\ \text { baixas }\end{array}\end{array}$

Escore 2

olhos fundos, sem

expressão,

desconfortável
Escore 3

olhos pálidos, pupilas dilatadas, ranger dentes, ganido, dorso encurvado, posição de proteção

*Devido à subjetividade das variáveis no pós-operatório, essa avaliação foi feita sempre pela mesma pessoa.

Fonte: Firth e Haldane (1999).

Houve diferença significativa da frequência cardíaca no M2 aos 120 minutos após a epidural. Em GC, GME e GM, a frequência manteve-se dentro dos parâmetros normais, porém, no GX, revertida aos 1440 minutos após a administração dos fármacos. A diminuição da frequência cardíaca no GX teve início em M2 e se prolongou até M4, sendo revertida totalmente após M5 (Fig. 1).

No GX, observou-se redução da frequência cardíaca em relação aos valores basais. Com relação às alterações fisiológicas e comportamentais, um animal nesse grupo (20\%) apresentou vômito 60 minutos após a administração do fármaco; houve alterações comportamentais em quatro animais $(80 \%)$ tais como: recusa de alimento, alienação em relação ao ambiente, mantendo as orelhas baixas e as pálpebras fechadas, e recusa para andar, urinar ou defecar. A administração de xilazina produziu alterações no ritmo respiratório, com maior profundidade respiratória no M2, normalizando-se no M5.

A administração do sulfato de morfina por via epidural provocou vômito em um $(20 \%)$ paciente após 30 minutos da injeção epidural, e três $(60 \%)$ animais do GM apresentaram prurido nos membros pélvicos e nas proximidades da ferida cirúrgica, decorridos 120 minutos. Em comparação ao grupo xilazina, dois (40\%) animais do GM apresentaram depressão respiratória, mas três $(60 \%)$ do GM não apresentaram alterações cardiorrespiratórias significativas durante todo o período de observação. Foi observado que as concentrações de cortisol plasmático apresentaram maiores valores no M2 e M3. Os animais do GM apresentaram menor valor 
de cortisol quando comparados ao do GC e ao GX no M2.

$\mathrm{Na}$ análise dos grupos, em relação à variável temperatura retal, o GME diferiu do GC no M2. Também houve diferença entre o GME e o GX no M3. Dentre todos os grupos, os animais do GM foram os que apresentaram menores valores de temperatura retal durante todo o período de observação.

Os valores de escore de comportamento diferiram $(\mathrm{P} \leq 0,05)$ entre os tratamentos empregados. Considerando-se individualmente o escore empregado, houve diferença significativa entre: a) ingestão de alimento - GME variou de forma significativa dos outros grupos: $\mathrm{GC}<\mathrm{GM}$ e $\mathrm{GX} ; \mathrm{b}$ ) postura - GC e GX diferiram de forma significativa do GM; c) aparência e manipulação - GC apresentou alterações significativas em relação aos GM, GME e GX. O GME não apresentou diferença significativa entre os momentos, permanecendo com valores estáveis durante as 24 horas de observação. Assim, observa-se que o efeito analgésico predominante está relacionado ao uso do opioide, constatado também pelos baixos valores do GM durante todos os momentos (Fig. 2).

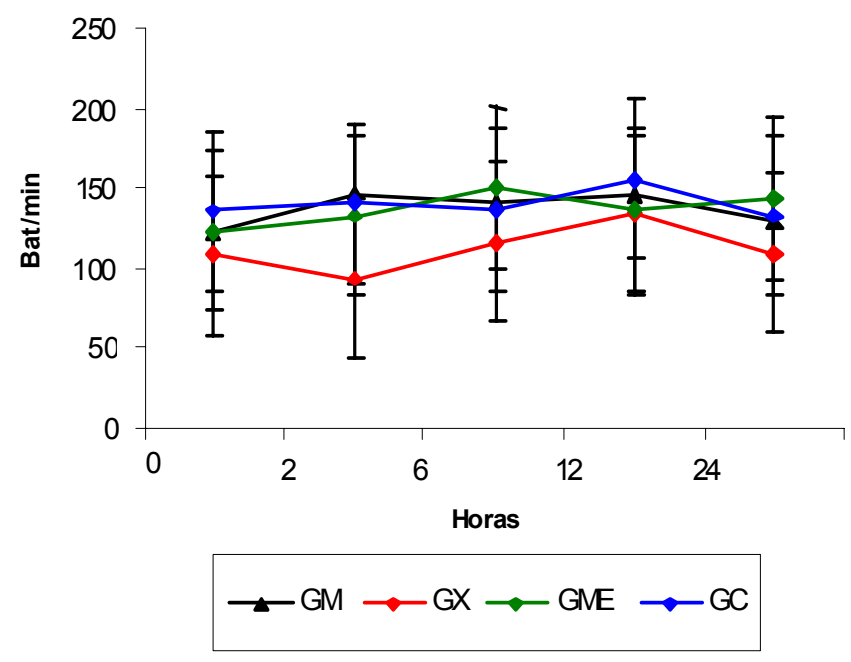

Figura 1. Variação média da frequência cardíaca (batimentos/minuto) em cães após tratamento epidural com morfina (GM), xilazina (GX), solução salina a $0,9 \%$ (GC) e meloxicam (GME).

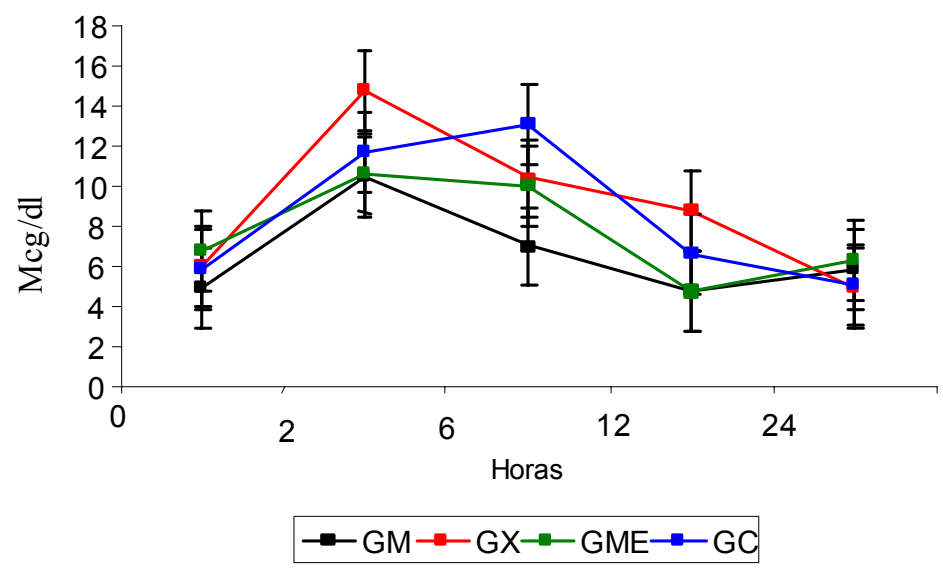

Figura 2. Variação média dos níveis de cortisol plasmático $(\mu \mathrm{g} / \mathrm{dL})$ em cães após tratamento epidural com morfina (GM), xilazina (GX), solução salina a $0,9 \%$ (GC) e meloxicam (GME). 
A bradicardia observada no GX provavelmente está relacionada ao efeito alfa-2 adrenoceptor agonista da xilazina, que pode culminar em bloqueio átrio-ventricular segundo Christovão et al. (2006). Em estudo semelhante, Kelawala et al. (1996) relataram decréscimo das frequências cardíaca e respiratória e da pressão arterial média após administração epidural de xilazina em cães. Antonaccio et al. (1973) afirmaram que a depressão da frequência cardíaca associada ao uso da xilazina se deve à acentuada ação sobre o tônus vagal. Para evitar os efeitos adversos associados aos fármacos alfa-2 agonistas, é recomendada a prévia administração de substâncias anticolinérgicas, exceto em cavalos e grande ruminantes.

Green e Thurmon (1988) ressaltaram os efeitos gastrintestinais associados à xilazina, entre eles salivação e relaxamento do esfíncter gastroesofágico, respectivamente em cães e ruminantes. A administração de xilazina por via subcutânea e intramuscular pode induzir vômito em cães e gatos, devido à ativação central do adrenorreceptor alfa- 2 de acordo com relatos de Hisaka et al. (1992).

Thurmon et al. (1996) relataram que os receptores opioides e alfa-2 são encontrados em regiões similares do cérebro e em alguns dos mesmos neurônios; ambas as ligações dos receptores opioides $m u$ e alfa-2 adrenérgicos resultam na ativação do mesmo sistema de sinal transdutor, ou seja, proteínas $G$ associadas à membrana. Essas proteínas induzem uma cascata de eventos que abrem os canais de potássio na membrana neuronal, denominados mecanismos efetores. A ativação dos canais de potássio nos neurônios pós-sinápticos causa à célula a perda de potássio, tornando-a hiperpolarizada; por este motivo, a célula se torna indiferente ao impulso excitatório.

De acordo com Thurmon et al. (1996) e Prado Filho et al. (2000), apesar de a xilazina por via epidural apresentar efeitos sistêmicos menos evidentes que por via parenteral, também observou-se efeitos depressores sobre o sistema cardiovascular em outras espécies. Os efeitos sistêmicos devem-se, possivelmente, à absorção do fármaco pela circulação. A ação dos alfa-2adrenoceptores sobre o sistema nervoso central inclui sedação, relaxamento muscular ou inibição da espasticidade, analgesia, ansiedade e efeitos anticonvulsivantes (Guimarães e Moraes, 2000; Kallas et al., 2001).

Com relação à perda dos reflexos observada nos animais do GX, já descrita por Tranquilli et al. (2005), o efeito sedativo e analgésico dessa substância ocorreu devido ao estímulo alfa 2, no cérebro, e à sua capacidade de bloquear a liberação de norepinefrina, consequentemente inibindo a transmissão interneural na medula espinhal, provocando o miorrelaxamento.

Outros efeitos podem ser observados com a administração intravenosa da xilazina segundo Caulkett et al. (1993), Cullen (1999) e Bagatini et al. (2002), tais como: a falta de resposta ao ambiente, rotação medial dos olhos e queda das pálpebras; distensão gástrica em cães, com recuperação prolongada, aumento na produção de urina por inibição da liberação do hormônio antidiurético, além do acréscimo na taxa de filtração glomerular.

Quanto à temperatura retal, Sessler e Ponte (1990) e Emerick et al. (1994) afirmaram que usualmente a TR permanece a mesma durante a anestesia epidural em cães, porém pode ocorrer hipotermia depois da injeção epidural, sendo resultado da redistribuição do calor no interior do corpo. A oscilação da temperatura na pele dos membros e a ausência de sudorese devem refletir mudanças na atividade simpática depois do bloqueio do nervo epidural.

Segundo Burford e Corley (2006), o anestésico local injetado por via espinhal no cão ingressa no líquído cerebroespinhal e no sistema linfático pelo processo de difusão através das veias durais linfáticas localizadas nas raízes nervosas, com o extravasamento da substância anestésica local para fora do canal vertebral por meio do forame intervertebral, ocorrendo a absorção vascular e a redistribuição sistêmica.

Yaksh e Rudy (1976) verificaram a presença de um grande número de receptores opioides na substância gelatinosa do corno dorsal da medula espinhal e sugeriram que a administração de pequenas doses de opioides no espaço epidural deve ser efetiva na produção de analgesia. Este fato foi confirmado pelos resultados obtidos neste trabalho em que a dose de $0,1 \mathrm{mg} / \mathrm{kg}$ de morfina, administrada no espaço peridural lombossacro das cadelas, garantiu maior grau de 
analgesia, tanto na intensidade quanto na duração.

Willians et al. (1993) garantiram bom embasamento para o uso de opioides via peridural, ao afirmarem que essa técnica, mesmo com doses menores, garante um grau de analgesia mais profundo e prolongado, quando comparado aos resultados obtidos pela administração do mesmo agente por via intramuscular ou intravenosa.

Relatos de Pascoe e Dyson (1990) explicam que os opioides administrados pela via epidural aliviam a dor somática e visceral pelo bloqueio seletivo dos impulsos nociceptivos sem interferir na função motora e sensorial ou deprimir o sistema nervoso simpático. Portanto, neste estudo observou-se que a administração de morfina ocasionou efeitos colaterais, entre eles: vômito, prurido e depressão respiratória.

Murphy (1993) e Greene et al. (1995) citaram que a eficácia do opioide é mais evidente se administrado antes do início da dor. Relataram, ainda, que o emprego de morfina epidural em cães apresentou baixos valores de escore de dor, concentrações de cortisol, adrenalina e noradrenalina, pressão sanguínea arterial sistólica e ritmos cardíaco e respiratório em relação a cães que receberam morfina intravenosa por um período de até 10 horas de pós-operatório. Os resultados desses autores foram semelhantes aos obtidos neste estudo com relação aos baixos valores de cortisol plasmático nos animais do grupo morfina.

A eficácia analgésica, a duração de ação e os efeitos adversos da morfina administrada via peridural também foram relatados por Lehmann (1997), Subramaniam et al. (2001), Valadão et al. (2002) e Silva et al. (2004). Esses efeitos foram sedação, bradicardia, depressão respiratória e vômito, comumente associados à administração sistêmica, comprovando o efeito analgésico mais intenso observado no GM. Thurmon et al. (1996) afirmaram que a morfina estimula diretamente o centro do vômito e os esfíncteres do trato gastrintestinal, podendo causar constipação, efeito adverso que pode ser equilibrado por meio do aumento do peristaltismo intestinal.
Cannon e Breedveld (2001) descreveram que os efeitos terapêuticos dos AINES decorrem da propriedade de interferir na síntese de prostaglandinas (PGs), por meio da inibição da ciclooxigenase (COX). Os AINES convencionais inibem as duas enzimas, COX-1 e COX-2, em consequência da redução das PGs envolvidas com mecanismos de proteção de alguns órgãos, sendo $o$ trato gastrintestinal o mais frequentemente acometido, seguido do sistema urinário e de discrasias sanguíneas, podendo causar efeitos adversos, como hemorragia submucosa, eritema, erosões superficiais, perda de sangue nas fezes, úlceras gástricas e duodenais e gastrite, segundo relatos de Costa et al. (2007).

De acordo com McCormack (1994), os antiinflamatórios não esteroidais possuem marcado efeito analgésico e não apresentam os efeitos colaterais citados dos opioides, como depressão respiratória e redução da motilidade gastrintestinal, e os efeitos psicomotores. Estas observações podem ser constatadas nos GM e GME durante o período de observação. No GME, não houve diferença significativa ente os momentos, quando comparado aos GM e GX, durante todo o período de observação.

Diferentemente do grupo tratado com morfina e meloxicam, quanto à frequência respiratória, $\mathrm{o}$ GX apresentou maior depressão cardiovascular e respiratória. No GME, a frequência respiratória e os valores de cortisol permaneceram estáveis durante todo $\mathrm{o}$ período de observação. Entretanto, os animais do GC mantiveram médias mais altas que as registradas nos GM, GX e GME. Leme et al. (2006) afirmaram que a resposta ao estresse agudo é acentuada pelas catecolaminas liberadas pela adrenal no sangue circulante, sendo possível verificar, neste estudo, que, nos GM, GX e GME houve redução dos valores de cortisol plasmático e escores de dor, quando comparados aos valores do grupocontrole.

\section{CONCLUSÕES}

A administração epidural de morfina em solução fisiológica $0,9 \%(0,3 \mathrm{~mL} / \mathrm{kg})$, em cães, produziu analgesia e sedação intensa, redução das concentrações de cortisol plasmático e menor incidência de efeitos colaterais no pós-operatório imediato. Bradicardia e diminuição da frequência 
respiratória foram observadas nos animais que receberam xilazina pela via epidural. $\mathrm{O}$ grau de analgesia foi considerado ineficiente com o uso de meloxicam quando comparado aos dos demais tratamentos.

\section{REFERÊNCIAS BIBLIOGRÁFICAS}

ANTONACCIO, M.J.; ROBSON, R.D.; KERWIN, L. et al. Evidence for increased vagal tone and enhancement of barorreceptor reflex activity after xylazine in anesthetized dogs. Eur. J. Pharmacol., v.23, p.311-315, 1973.

BAGATINI, A.; GOMES, C.R.; MASELLA, M.Z. et al. Dexmedetomidine: pharmacology and clinical application. Rev. Bras. Anestesiol., v.52, p.606-617, 2002.

BURFORD, J.H.; CORLEY, K.T.T. Morphineassociated pruritus after single extradural administration in a horse. Vet. Anaesth. Analg., v.33, p.193-198, 2006.

CANNON, G.W.; BREEDVELD, F.C. Efficacy of ciclooxygenase-2 specific inhibitors. Am. J. Med., v.110, suppl. 3A, p.6S-12S, 2001.

CAUlKeTT, N.; CRIBB, P.H.; DUKE, T. Xylazine epidural analgesia for cesarean section in cattle. Can. Vet. J., v.34, p.674-676, 1993.

COSTA, P.R.S.; ARAUJO, R.B.; COSTA, M.C. et al. Gastroduodenal endoscopy after nimesulide, monophenylbutazone and meloxicam administration in dogs. Arq. Bras. Med. Vet. Zootec., v.59, p.903-909, 2007.

CULLEN, L.K. Xylazine and medetomidine in small animals: these drugs should be used carefully. Aust. Vet. J., v.77, p.722-723, 1999.

CHRISTOVÃO, F.G.; ZAMUR, G.; MATAQUEIRO, M.I. et al. Sedative and antinociceptive effects of romifidine and xylazine in Thoroughbred mares. Arq. Bras. Med. Vet. Zootec., v.58, p.1030-1036, 2006.

DER G.; EVERITTY, B.S. A handbook of statistical analyses using SAS. 2.ed. Boca Raton: Chapman \& Hall, CRC Press, 2002. 364p.

EMERICK, T.H.M.D.; OZAKI, M.M.D.; SESSLER, D.I.M.D. et al. Epidural anesthesia increases apparent leg temperature and decreases the shivering threshold. Anesthesiology, v.81, p.289-298, 1994.
FANTONI, T.D.; CORTOPASSI, G.R.S. Anestesia em cães e gatos. São Paulo: Roca, 2002. p.199-208, 321-335.

FIRTH, A.M.; HALDANE, S.L. Development of a scale to evaluate postoperative pain in dogs. $J$. Am. Vet. Med. Assoc., v.214, p.651-659, 1999.

FIERHELLER, E.E.; CAULKET, N.A.; BAILEY, J.V. et al. A romifidine and morphine combination for epidural analgesia of the flank in cattle. Can. Vet. J., v.45, p.917-923, 2004.

GREEN, S.A.; THURMON, J.C. Xylazine: a review of its pharmacology and use in veterinary medicine. J. Vet. Pharmacol. Ther., v.11, p.295313, 1988.

GREENE, S.A.; KEEGAN, R.D.; WEIL, A.B. Cardiovascular effects after epidural injection of xylazine in isoflurane-anesthetized dogs. Vet. Surg., v.24, p.283-289, 1995.

GUIMARAES, L.D.; MORAES, A.N. Anesthesia in birds: anesthetics agents. Cienc. Rural, v.30, p.1073-1081, 2000.

HISAKA, Y.; OGASAWARA, S.; TASKASE, K. Alpha adrenoceptor subtypes involved in the emetic action of dogs. J. Pharmacol. Exp. Ther., v.261, p.746-754, 1992.

KALLAS, E.; SCHNAIDER, T.B.; JULIANO, Y. et al. Modelo de anestesia em coelhos para procedimentos no tórax. Acta. Cir. Bras., v.16, p.91-96, 2001.

KELAWALA, N.H.; KUMAR, A.; CHAUDARY, S. et al. Effects of epidural xylazine with diazepam premedication in dogs. Indian Vet. J., v.73, p.552-557, 1996.

LEHMANN, K.A. Tramadol in acute pain. Drugs, v.53, p.25-33, 1997.

LEME, F.O.P.; WURZINGER, L.J.; VASCONCELOS, A.C. et al. Platelets activation in equine with induced laminits and treated with ketoprophen, phenylbutazone and flunixin meglumine. Arq. Bras. Med. Vet. Zootec., v.58, p.149-157, 2006.

McCORMACK, K. Non-steroidal antiinflamatory drugs and spinal nociceptive processing. Pain., v.59, p.09, 1994.

MURPHY, D.F. Interpleural analgesia. $B r . J$. Anaesth., v.71, p.426-434, 1993. 
OLESKOVICZ, N. Injeção epidural de cetamina ou cetamina levógira no controle da dor pósincisional em equinos. 2001. Dissertação (Mestrado) - Faculdade de Ciências Agrárias e Veterinárias, Universidade Estadual Paulista, Jaboticabal.

PASCOE, P.J.; DYSON, D.H. Postoperative analgesia following lateral thoracotomy: epidural morphine $v s$ intercostals bupivacaine. In: Annual SCIENTIFIC MEETING OF THE AMERICAN COLLEGE OF VETERINARY ANESTHESIOLOGISTS, 30., 1990, Middleburg. Proceedings... Middleburg, VA: ACVA, 1990.

PRADO FILHO, O.R.; STEFFENS, V.A.; SANTOS, A.B. et al. xilazina como prémedicação para anestesia com tiopental sódico em cães. Acta Cir. Bras., v.15, 2000. Disponível em: $<$ http://www.scielo.br/>. Acessado em: 07 Mai. 2008.

SESSLER, D.I.; PONTE, J. Shivering during epidural anesthesia. Anesthesiology, v.72, p.816821, 1990.

SILVA, O.C. Analgesia peridural em bovinos através do emprego da associação de morfina e lidocaina. 1997. 69f. Dissertação (Mestrado) Faculdade de Ciências Agrárias e Veterinárias, Universidade Estadual Paulista, Jaboticabal.
SILVA, N.S.F.; SAKATA, R.K.; ISSY, A.M. et al. Correlation between CSF concentration and side effects after spinal morphine injection in rats. Rev. Bras. Anestesiol., v.54, p.53-59, 2004.

SUBRAMANIAM, B.; SUBRAMANIAM, K.; PAWAR, D.K. et al. Preoperative epidural ketamine in combination with morphine does not have a clinically relevant intra- and postoperative opioid-sparing effect. Anesth. Analg., v.93, p.1321-1326, 2001.

TRANQUILLI, W.J.; GRIMM, K.A; LAMONT, L.A. et al. Tratamento da dor para o clínico de pequenos animais. São Paulo: Roca, 2005. p.912.

THURMON, J.C.; TRANQUILLI, J.W.; BENSON, J.G. Lumb \& Jones - Veterinary anesthesia. New York: Lea \& Febiger, 1996. v.3, p.187-203, 434-445.

VALADÃO, A.A.C.; DUQUE, C.J.; FARIAS, A. Administração epidural de opioides em cães. Cienc. Rural, v.32, p.347-355, 2002.

WILLIANS, L.L.; BOUDRIEAU, R.J.; CLARK, G. et al. Evaluation of epidural morphine in dogs for pain relief after hindlimb orthopedic surgery. Vet. Surg., v.22, p.89, 1993.

YAKSH, T.L.; RUDY, T.A. Analgesia mediated by a direct spinal action of narcotics. Science, v.192, p.1357-8, 1976. 